Check for updates

Cite this: RSC Adv., 2020, 10, 20784

Received 26th April 2020

Accepted 11th May 2020

DOI: $10.1039 / \mathrm{dOra03763j}$

rsc.li/rsc-advances

\section{Recent advances in the synthesis of biologically and pharmaceutically active quinoline and its analogues: a review}

\begin{abstract}
Abdanne Weyesa and Endale Mulugeta (D)*
Recently, quinoline has become an essential heterocyclic compound due to its versatile applications in the fields of industrial and synthetic organic chemistry. It is a vital scaffold for leads in drug discovery and plays a major role in the field of medicinal chemistry. Nowadays there are plenty of articles reporting syntheses of the main scaffold and its functionalization for biological and pharmaceutical activities. So far, a wide range of synthesis protocols have been reported in the literature for the construction of this scaffold. For example, Gould-Jacob, Friedländer, Pfitzinger, Skraup, Doebner-von Miller and Conrad-Limpach are well-known classical synthesis protocols used up to now for the construction of the principal quinoline scaffold. Transition metal catalysed reactions, metal-free ionic liquid mediated reactions, ultrasound irradiation reactions and green reaction protocols are also useful for the construction and functionalization of this compound. The main part of this review focuses on and highlights the above-mentioned synthesis procedures and findings to tackle the drawbacks of the syntheses and side effects on the environment. Furthermore, various selected quinolines and derivatives with potential biological and pharmaceutical activities will be presented.
\end{abstract}

\section{Introduction}

Quinoline is the most ubiquitous heterocyclic aromatic compound with a potential for industrial and medicinal

Department of Applied Chemistry, School of Applied Natural Science, Adama Science and Technology University, P. O. Box: 1888, Adama, Ethiopia. E-mail: endale. mulugeta@astu.edu.et applications. 1-Azanapthalene and benzo[b]pyridine are used as alternative names for quinoline (Fig. 1).

It has a characteristic double-ring structure containing a benzene ring fused with a pyridine moiety, with the molecular formula $\mathrm{C}_{9} \mathrm{H}_{7} \mathrm{~N}^{1}{ }^{1}$ Quinoline is an essential segment of both natural and synthetic compounds. In particular, the pyranoquinoline ring system has gained considerable attention as

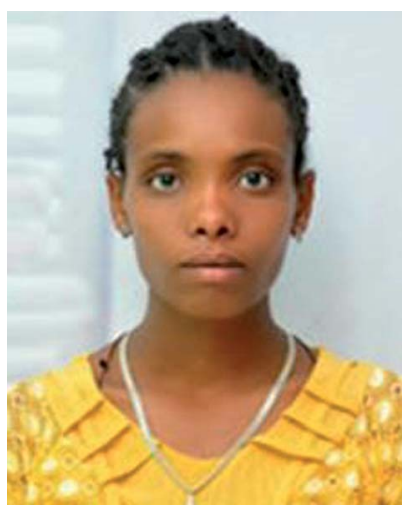

Abdanne Weyesa was born in Wolisso, Ethiopia. She graduated from Adama Science and Technology University where she remained to carry out her $\mathrm{MSc}$ studies under the supervision of Dr Endale Mulugeta. Her research focuses on the synthesis of bioactive chalcone derivatives anchored with heterocyclic compounds in different classes that have pharmacological activities.

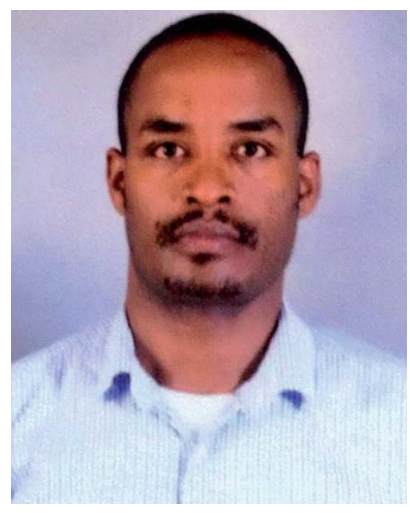

Endale Mulugeta received his MSc from the College of Natural and Computational Sciences, Department of Chemistry, Addis Ababa University, Ethiopia in 2010 and his PhD from the College of Natural Sciences, Department of Chemistry, Kangwon National University, South Korea in 2018. He joined Adama Science and Technology University as an Assistant Professor of Organic Chemistry in 2018. His research topics include the study and development of new methods and synthetic approaches towards organic compounds, as well as a focus on developments in the synthesis of heterocyclic organic compounds of different classes that have pharmacological activity. 


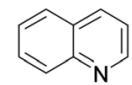

Quinoline 1

Fig. 1 Chemical structure of quinoline.

it is a core structure, constituting the basic skeleton of a number of alkaloids. ${ }^{2}$ Generally quinoline is present in pharmacologically active natural products and in synthetic products. This compound is used mainly as a central template for the synthesis of various drugs. Quinoline is a weak tertiary base and can form salts with acids. It exhibits similar reactions to pyridine and benzene and can also participate in both electrophilic and nucleophilic substitution reactions. It is nontoxic to humans. ${ }^{3}$ For the construction and functionalization of this noble compound and its derivatives, an enormous number of synthesis techniques have been reported, among which conventional or classical methods, transition metal free catalysed methods, ultrasound irradiation reactions and greener chemical processes have been well explored. Most of the time scholars explore classical reaction methodologies, such as Gould-Jacobs, ${ }^{4}$ Friedländer, ${ }^{5}$ Pfitzinger, ${ }^{6}$ Skraup, ${ }^{7}$ Doebner-von Miller, ${ }^{8}$ and Conrad-Limpach, ${ }^{9}$ and modify them with ecofriendly transition metal mediated, ultrasound irradiation reactions or greener protocols. ${ }^{10,11}$

Currently, studies revealing numerous natural products and synthetic derivatives incorporating a quinoline scaffold have attracted scholars' attention because they exhibit a broad range of biological and pharmaceutical activities.

For example antibacterial, antioxidant, anticancer, antiinflammatory, antimalarial, antifungal and antileishmanial activities have been well studied. ${ }^{12}$ Shang and co-workers reported a comprehensive review on alkaloids with a quinoline moiety as a core scaffold, isolated from compounds from natural sources and showing bioactivity potential. ${ }^{13}$ The review comprehensively organized into two focal sections. First various synthesis strategies will be addressed to highlight the original reaction procedures and modifications in the recent literature related to all the synthetic strategies accordingly. Then novel pharmaceutically and biologically active quinolines will be explored (Fig. 2).

\section{Synthesis of quinoline and its derivatives}

So far scholars have explored copious synthesis protocols to construct and functionalize the quinoline scaffold. Here, we focus on a review of the classical synthesis procedures named GouldJacob, Friedländer, Pfitzinger, Skraup, Doebner-von Miller, and Conrad-Limpach reactions, transition metal catalysed reactions, transition metal free ionic liquid mediated reactions, ultrasound irradiation reactions, and greener chemical processes for the synthesis of quinoline and its analogues will be discussed briefly.

\subsection{Gould-Jacob quinoline synthesis}

Various quinoline scaffolds with substituents at carbon- 4 can be prepared through cascade reactions known as the Gould-Jacob

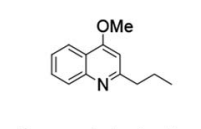

2-n-propylquinoline 2
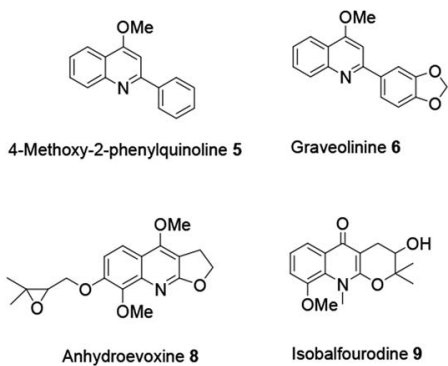

Isobalfourodine 9

Chimanine B 3

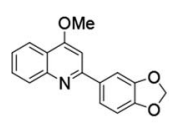

Graveolinine 6

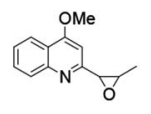

Chimanine D 4

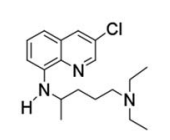

Chloroquine 11

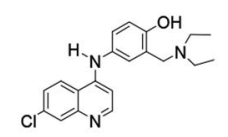

Amodiaquine 12

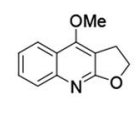

Dictamnine 7

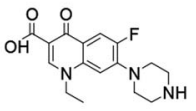

Norfloxacin 10

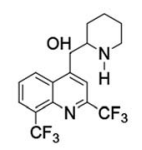

Mefloquine 13

Fig. 2 Structures of bioactive compounds from natural sources.

cyclization reaction. ${ }^{4}$ In this procedure 4-hydroxyquinoline $\mathbf{1 6}$ is prepared from aniline (14) and diethyl ethoxymethylenemalonate (15) involving series of reactions to provide quinoline 16 (Scheme 1).

This protocol helps in the preparation of various commercially available drugs based on a quinoline scaffold as the core skeleton. For instance, the preparation of the well-known nonsteroidal anti-inflammatory drugs floctafenine and glafenine relies on this synthesis methodology. ${ }^{4}$

\subsection{Friedländer quinoline synthesis}

In this procedure ortho-substitution of aniline $\mathbf{1 7}$ and aldehyde or ketone $\mathbf{1 8}$ with a reactive $\alpha$-methylene group via condensation followed by cyclodehydration reactions affords compound 19 (Scheme 2). In this reaction procedure, regioselectivity is a challenging issue when unsymmetrical ketones are used. ${ }^{5}$ The reaction is well catalysed using a base or acid, such as a Brønsted acid or a Lewis acid, and ionic liquids can also activate the reaction well. Furthermore, it can proceed smoothly without a catalyst by heating the mixture. The merit of this reaction procedure is the scope of substrates of various functional groups that are well tolerated on both arylamine and ketone moieties.

\subsection{Modified Friedländer quinoline synthesis}

Anand and co-workers operationally developed a simple, highly efficient, practical and convenient one-pot method for the synthesis of a broad scope of quinoline derivatives. ${ }^{6}$ Here, by

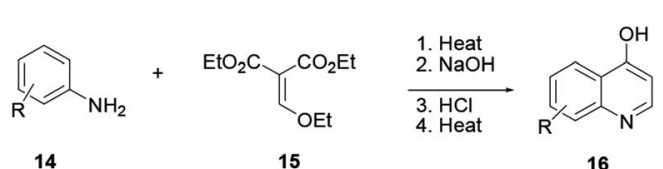

Scheme 1 General scheme of the Gould-Jacobs quinoline synthesis. 


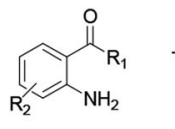

17

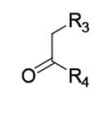

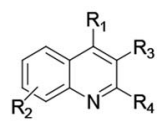

19
Scheme 2 General reaction scheme of Friedländer quinoline synthesis.

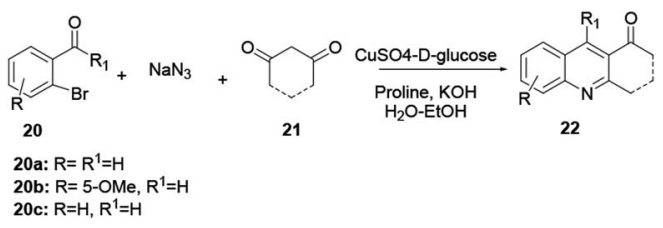

Scheme 3 General reaction scheme of modified Friedländer quinoline synthesis.

using 2-bromobenzaldehyde (20), acyclic or cyclic 1,3-diketone (21) and sodium azide in a three-component reaction protocol in the presence of an air-stable, eco-efficient and inexpensive catalyst, quinoline $\mathbf{2 2}$ is prepared in good yields. In this reaction copper salt-D-glucose helps to generate $\mathrm{Cu}(\mathrm{I})$ species in situ through reduction in aqueous ethanol as a green solvent, and proline is used as a ligand and proton source to synthesize the target compound (Scheme 3). This protocol follows an Ullmanntype coupling reaction where nucleophilic substitution of $\mathrm{Br}$ from 2-bromobenzaldehyde (20) with sodium azide gives an azido complex intermediate. The azido-Cu complex is subjected to reductive elimination followed by dehydrative cyclocondensation, providing the desired quinoline 22.

The authors claim that this method worked nicely with both electron-donating and electron-withdrawing substituent groups at the ortho-, meta-, and para-positions of the phenyl ring. ${ }^{6}$

\subsection{Pfitzinger quinoline synthesis}

This procedure is also known as the Pfitzinger-Borsche reaction. Here isatin $\mathbf{2 3}$ reacting with $\alpha$-methylene carbonyl compound 24 in the presence of a base in ethanol provides substituted quinoline derivative 25 (Scheme 4). ${ }^{6}$ In this reaction protocol, isatic acid is formed from isatin 23 and condensed with $\alpha$-methylene carbonyl compound $\mathbf{2 4}$ in the presence of a strong base. Subsequent decarboxylation affords quinoline $\mathbf{2 5}$. This procedure is an extension of the Friedländer quinoline synthesis protocol. The reaction basically depends on the more stable isatin varieties instead of the ortho-aminoaryl moieties that are the basic starting materials for the preparation of quinoline via the former reaction protocol. ${ }^{7}$ Elghamry and coworkers used a similar reaction protocol with minor

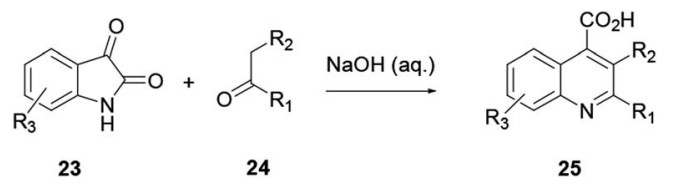

Scheme 4 General reaction scheme of the Pfitzinger quinoline synthesis.

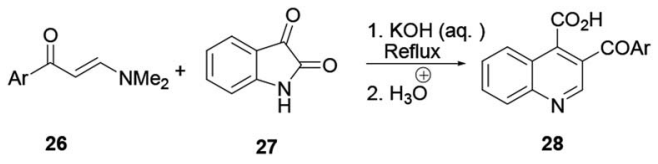

Scheme 5 Synthesis of quinoline derivative 28 through the Pfitzinger reaction.

modifications in a one-step and one-pot method to synthesize quinoline-4-carboxylic acids 28 in water (Scheme 5). Isatin (27) was refluxed with enaminone 26 in the presence of an aqueous solution of $\mathrm{KOH}$ or $\mathrm{NaOH}$, and subsequent acidification using dilute $\mathrm{HCl}$ to prepare quinoline-4-carboxylic acid (28) in good to excellent yields. ${ }^{7}$ The authors claimed that using enaminone as a replacement for 1,3-dicarbinols improves the yield and practicality of the reaction.

\subsection{Skraup/Doebner-von Miller quinoline synthesis}

A synthesis of quinoline via aniline and glycerine in the presence of a strong acid and an oxidant under reflux was revealed by Skraup and co-workers. ${ }^{8}$ Here, a crotonaldehyde intermediate is generated in situ from glycerol $\mathbf{3 0}$. Subsequently aniline 29 is added to the reaction under heating to provide quinoline 31. The reaction of substituted acrolein 32 with aniline 29 in the presence of an oxidant to provide quinoline $\mathbf{3 3}$ is known as the Doebner-von Miller protocol (Scheme 6).

The fundamental drawbacks of the Skraup and Doebner-von Miller syntheses are that both turn out to be violently exothermic during the progress of the reaction, and the variety of oxidants and the highly acidic medium required make isolation of the desired product tedious. Regioselectivity is also a concern when meta or 3,4-disubstituted anilines are used..$^{9}$ 2methylquinoline and its derivatives have shown substantial biological activities. However, there are different techniques for the synthesis of 2-methylquinoline, and Doebner-von Miller is the best. Yalgin and co-workers report the synthesis of 2-methylquinoline 36 using a modified Doebner-von Miller reaction protocol in the presence of a strong acid in a flow reactor with aniline and acrolein. ${ }^{15}$ 2-Methylquinoline derivative $\mathbf{3 6}$ synthesized using a continuous flow in water through Doebner-Miller reaction

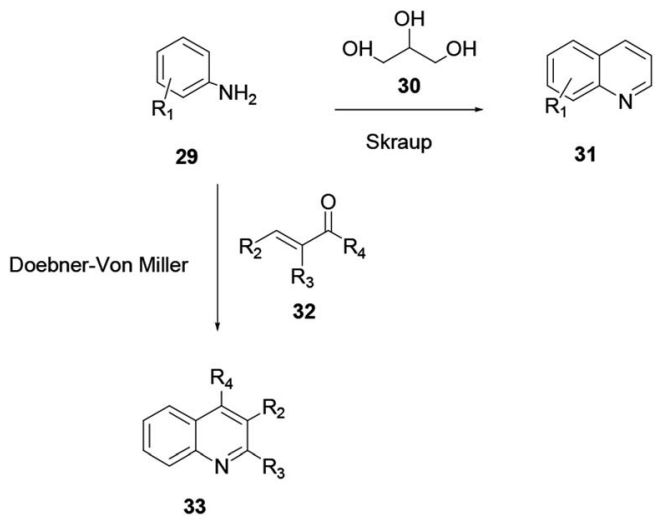

Scheme 6 General reaction scheme of Skraup/Doebner-von Miller quinoline synthesis. 


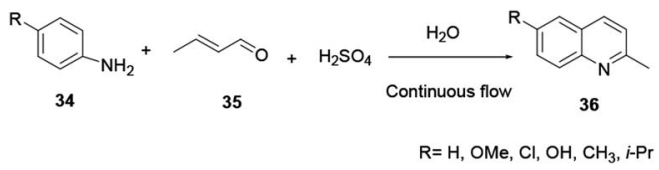

Scheme 7 General reaction scheme of Doebner-von Miller quinoline synthesis.

procedure. This method is a rapid and green route for the synthesis of quinoline derivatives to provide a good to excellent yields (Scheme 7). ${ }^{\mathbf{1 4}}$

\subsection{Combes/Conrad-Limpach quinoline synthesis}

The Combes/Conrad-Limpach reaction is the condensation of a primary aryl amine with a 1,3-diketone or $\beta$-keto-aldehyde or 1,3-dialdehyde, giving an enamine intermediate which is further heated in a strong acid, and later cyclodehydration to afford quinoline. Here, the condensation of primary aryl amine 37 with $\beta$-diketone $\mathbf{4 0}$ in acid catalysis, followed by ring closure of a Schiff base, affords quinoline $\mathbf{4 1}$ (Scheme 8). ${ }^{\mathbf{1 0}}$

Using the same reaction procedure the Conrad-Limpach scheme can be used to prepare various quinoline derivatives using $\beta$-ketoester 38 in place of $\beta$-diketone $\mathbf{4 0}$. Both procedures follow the condensation of arylamine with $\beta$-ketoester or $\beta$ diketone by a further cyclodehydration reaction and heating in strong acid to prepare the target compounds.

\subsection{Ultrasound irradiation reactions}

The reaction time, product yields and qualities of this reaction procedure are better than those from the above-mentioned quinoline synthesis protocols. It is one of the greener quinoline synthesis protocols. Using an ultrasound irradiation reaction procedure via two sequential reactions, $\mathrm{S}_{\mathrm{N}} 2$ followed by a condensation reaction, quinoline 45 can be synthesized in good yield (Scheme 9). ${ }^{\mathbf{1 2}}$

This procedure has the advantages of a short reaction time and easy isolation of the product and provides good-to-excellent

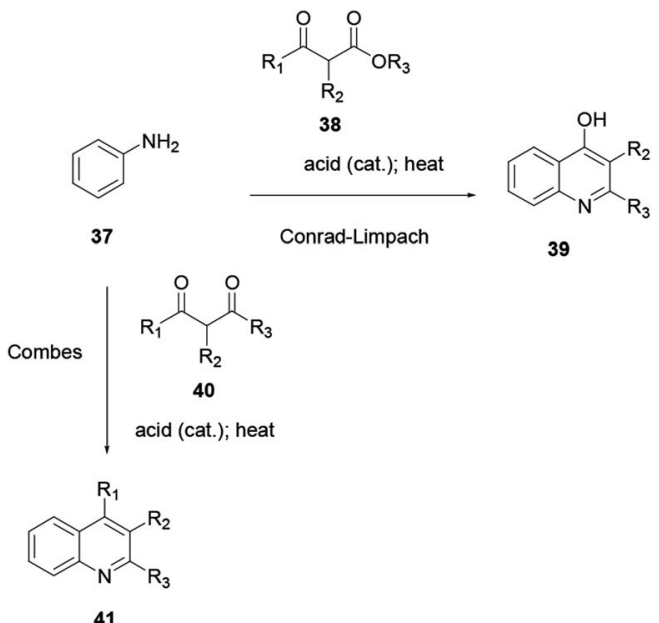

Scheme 8 General reaction scheme of the Combes/Conrad-Limpach quinoline synthesis.

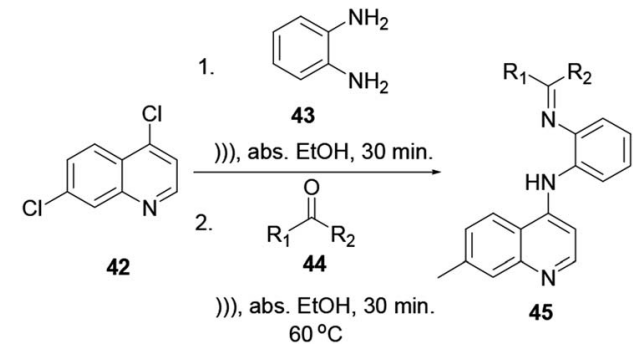

Scheme 9 Synthesis of a quinoline derivative using ultrasound irradiation.

yields. ${ }^{16}$ Quinoline 45 can be easily functionalized at the 4chloro position with $o$-phenylenediamine $\mathbf{4 3}$, and further reacted with unsymmetrical ketone $\mathbf{4 4}$ via a green chemistry protocol to provide quinoline derivative $\mathbf{4 5}$.

Xie and co-workers report C2-sulfonation of $\mathrm{N}$-oxide quinoline (46) using sulfonyl chloride 47 in the presence of Zn-dust, with water as a solvent via an ultrasound-assisted protocol, providing quinoline $\mathbf{4 8}$ in moderate yield. ${ }^{17}$ Here, sulfonyl chloride $\mathbf{4 7}$ is first reduced using $\mathrm{Zn}$-dust. The generated intermediate is coordinated with $N$-oxide quinoline 46. Finally, the target compound $\mathbf{4 8}$ is synthesized through an intramolecular nucleophilic addition reaction (Scheme 10).

\subsection{Transition metal free quinoline synthesis}

Various scholars report the synthesis of quinoline and its derivatives via metal-free mediated reaction protocols, such as ionic liquid, simple acid or base catalyst, using molecular iodine and catalyst-free reactions. The above-mentioned procedures are considered to be green chemical processes. Conducting reactions using the above strategies can achieve a high level of atom efficiency. The reaction media are recyclable, they provide high yields, have a short reaction time, are practical to operate under mild reaction conditions, conduct the reduction in a number of steps, decrease waste and are ecofriendly. ${ }^{18}$ Here, two amine derivatives, enamide 49 and imine $\mathbf{5 0}$, react in the presence of iodine in air to afford quinoline $\mathbf{5 1}$ (Scheme 11). In order to improve the reaction efficiency, various types of catalysts were explored. Among all these catalysts, iodine exhibited the highest yields. Moreover, the reaction has attracted a great deal of attention with its benefits of low toxicity, ability to operate under mild reaction conditions, lowcost starting materials and broad scope of substrates.

The three-component reaction of methyl ketone 52, arylamine 53, and $\alpha$-ketoester 54 in the presence of iodine and a catalytic amount of hydroiodic acid provides quinoline 55. In this reaction the $\mathrm{HI}$ co-product acts as a promoter with good

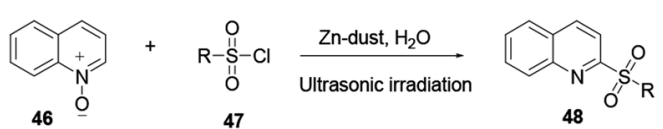

Scheme 10 Synthesis of a quinoline derivative using a non-metal catalyst. 


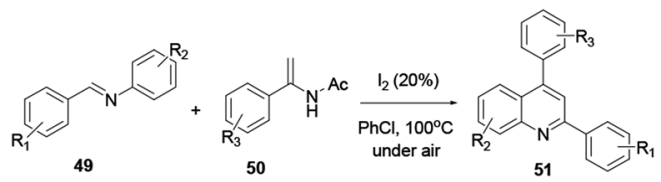

Scheme 11 Synthesis of a quinoline derivative using a non-metal catalyst.

$$
\text { (1) }
$$

Scheme 12 Three-component iodine-catalysed quinoline synthesis

functional compatibility (Scheme 12). ${ }^{19}$ Under transition metal free reaction conditions oxygen acts as an oxidant, where 2(aminomethyl)-aniline 56 reacts with aromatic ketone $\mathbf{5 7}$ to provide the corresponding quinoline $\mathbf{5 8}$ with excellent yield (Scheme 13). Wu and co-workers reported that the reaction procedure for the synthesis of quinoline can be achieved under aerobic reaction conditions without the presence of a catalyst, with lithium chloride salt additive in dimethylacetamide solvent. ${ }^{20}$ Following this reaction procedure ketones bearing strong electron-withdrawing or electron-donating groups could react smoothly and in an eco-friendly way to provide the desired product.

Carbaldehyde 59 reacted with substituted aniline 29 without a catalyst to provide quinoline $\mathbf{6 0}$ in excellent yield (Scheme 14). ${ }^{21}$ The reaction is performed at room temperature, with a short reaction time, providing excellent yield with no catalyst required (Scheme 14).

Through a convenient two consecutive steps ethyl-4-hydroxy2-methylquinoline-3-carboxylate (63) was synthesized. Using solid triphosgene in THF, commercially available 2-aminobenzoic acid 61 converted to isatoic anhydride 62, reacted with the sodium enolate of ethyl acetoacetate in warm DMA, to afford substituted quinoline 63 (Scheme 15). ${ }^{22}$

Zhu and co-workers reported an environmentally friendly reaction methodology to prepare quinoline $66^{23}$ In this reaction procedure the cheap and commercially available material 2nitrobenzyl alcohol (64) reacted with ketone 65 via an intramolecular redox process to afford synthetically important quinoline 66 in moderate yields (Scheme 16).

2-Sulfonylquinoline 69 was prepared smoothly without a base or organic solvent from quinoline $N$-oxide $\mathbf{4 6}$ and sodium sulfinate $\mathbf{6 7}$ using a metal-free and dual radical coupling reaction protocol devised by Xie and co-workers. ${ }^{24}$ Sodium sulfinate

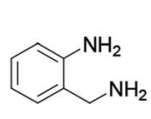

56

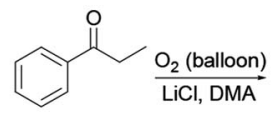

57

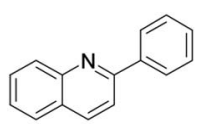

58
Scheme 13 Metal-free, aerobic quinoline synthesis.

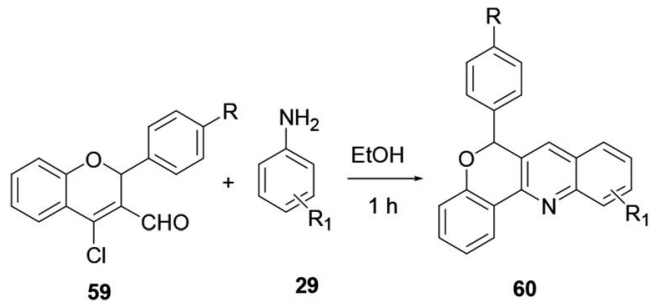

Scheme 14 Synthesis of quinoline derivative 60

is well-known for use as a sulfonating reagent in the following reactions, because it is stable to moisture and is safe and easy to handle. Additionally, Han and co-workers report the synthesis of 2-sulfonylquinoline 69 using quinoline $\mathrm{N}$-oxide with sodium sulfinate as starting materials in the presence of $\mathrm{K}_{2} \mathrm{~S}_{2} \mathrm{O}_{8}$ as an oxidant under an inert atmosphere in a mixed solvent system. ${ }^{25}$ Peng and co-workers amended previously reported protocols and reported the synthesis of 2-sulfonylquinoline 69 from quinoline $N$-oxide and sodium sulfinate via an easy, metal-free, oxidant-free and solvent-free method under mild reaction conditions using $\mathrm{TsCl}$ in water. ${ }^{26}$ Furthermore, 2-sulfonylquinoline 69 was synthesized from quinoline $N$-oxide and sulfonic acid 68 using organic dye as a catalyst (Scheme 17). ${ }^{27}$

The reaction was performed with a greener process protocol, and in ambient air to provide good to excellent yields.

\subsection{Transition metal mediated protocols}

$\mathrm{Xu}$ and co-workers reported the one-pot synthesis of substituted quinoline $\mathbf{7 1}$ with a broad scope of substrates using a transition $\mathrm{Co}(\mathrm{III})$ metal mediated reaction protocol. ${ }^{28} \mathrm{Co}$ (III) catalysis via a cascade of reactions, C-H activation, carbonylation and cyclization of aniline $\mathbf{2 9}$ and ketone $\mathbf{7 0}$ with paraformaldehyde provide various useful quinoline derivatives (Scheme 18). Benefits of the procedure are the broad scope of substrates with tolerance to various functional groups and the affordance of good to excellent yields. Moreover, it releases water and hydrogen gas as by-products.

Das and co-workers reported the synthesis of various polysubstituted quinolines $\mathbf{7 5}$ from commercially available $\alpha$ -

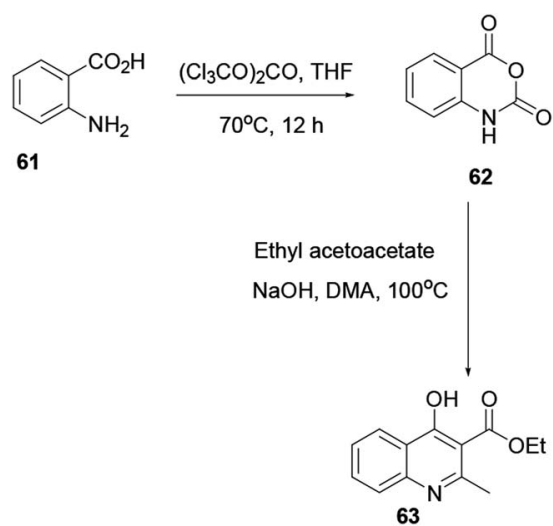

Scheme 15 Synthesis of quinoline derivative 63 


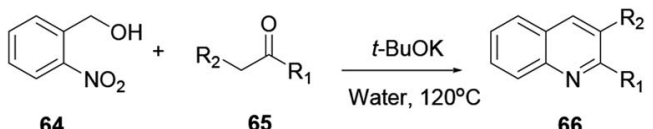

Scheme 16 Synthesis of quinoline 66 in water.

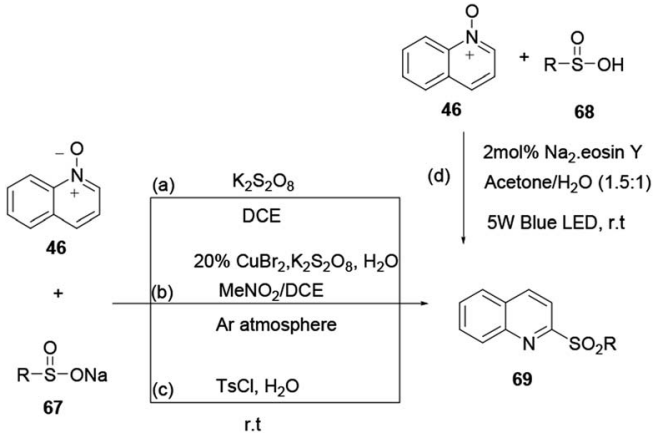

Scheme 17 Synthesis of quinoline 69 through various reaction conditions.

aminoaryl alcohol 72 and acyclic/cyclic ketones 73 or secondary alcohol 74 via a sequential dehydrogenation and condensation reaction process (Scheme 19). ${ }^{29}$ The advantage of conducting reactions via this method is that it uses a simple-to-handle and inexpensive nickel(II) catalyst. Luo and co-workers reported 6chloro-2-dimethyl-4-phenylquinoline, an antiparasitic agent, and 3,4-diphenylquinoline-2(1H)-one, a p38aMAP kinase inhibitor, as biologically active quinoline derivatives. The reaction proceeds through a single-step process and under neutral reaction conditions using a copper triflate catalyst in nitromethane. ${ }^{30}$ In contrast to an acid-catalysed two-step $[4+2]$ cycloaddition and oxidation reaction, it allows a single-step reaction to provide quinoline in high yields and excellent regioselectivity (Scheme 20). $N$-(2-alkenylaryl)enamine 79 is a strategic precursor for the synthesis of quinoline $\mathbf{8 0}$ or $\mathbf{8 1}$ using a one-pot copper-catalysed aerobic oxidative cyclization

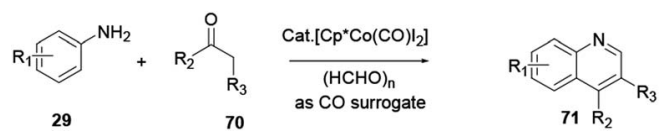

Scheme 18 Cobalt-catalysed synthesis of quinoline derivatives.

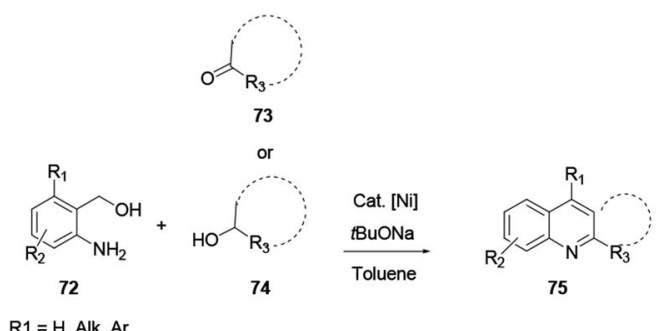

Scheme 19 Nickel-catalysed synthesis of polysubstituted quinolines.

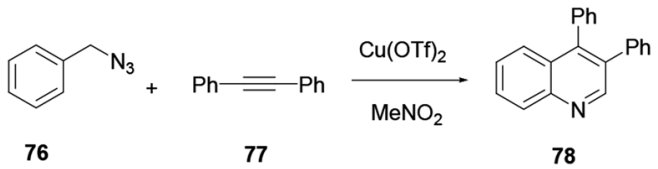

Scheme 20 Copper(I) triflate mediated synthesis of a quinoline derivative.

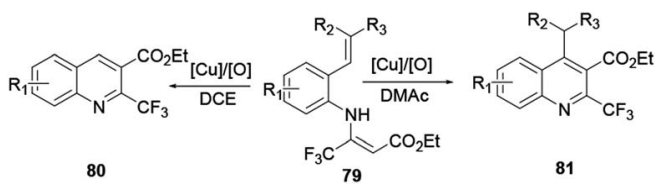

Scheme 21 Synthesis of 2-trifluoromethylquinoline.

reaction (Scheme 21). Chen and co-workers reported a novel and efficient procedure for the synthesis of 2-trifluoromethylquinolines $\mathbf{8 0}$ and $\mathbf{8 1}$ using copper-chloride salt under aerobic reaction conditions with various functional group tolerances. ${ }^{31}$

2,3-Disubstituted quinoline $\mathbf{8 4}$ was synthesized through multicomponent coupling reactions of alkyne 83, amine 29 and aldehyde 82 using a zinc(II) triflate salt catalyst (Scheme 22). ${ }^{32}$ The merits of this procedure are that reactions can proceed without the presence of ligands or co-catalysts, under solventfree and inert reaction conditions. Bao and co-workers recently reported a simple and efficient synthetic protocol for the construction of sulfonylated quinoline in water via a zinc powder mediated coupling reaction. ${ }^{33}$

Haloquinolines 85 and $\mathbf{8 6}$ reacted with sulfonyl chloride in water in the presence of cheap metal zinc powder to afford sulfonylated quinolines $\mathbf{8 7}$ and $\mathbf{8 8}$, respectively (Scheme 23).

Mondal and co-workers reported a neat $\mathrm{Zn}^{\mathrm{II}} / \mathrm{Cu}^{\mathrm{I}}$ catalyzed reaction procedure to synthesise quinoline 91 through the uncommon $\mathrm{sp}^{2} \mathrm{C}-\mathrm{H}$ activation of three-component protocols. ${ }^{34}$ Aniline 29, alkynes 89 and aldehydes 90 react via a cascade cyclization reaction to provide quinoline 91 (Scheme 24).

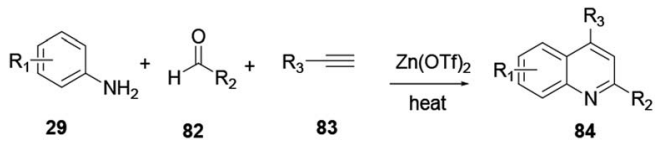

Scheme 22 Zinc triflate mediated synthesis of quinoline derivatives.

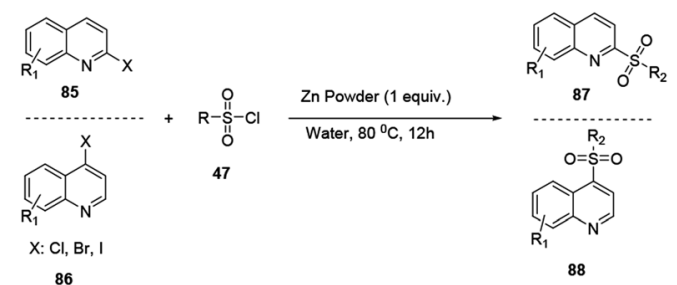

Scheme 23 Zinc powder mediated synthesis of sulfonylated quinoline derivatives. 


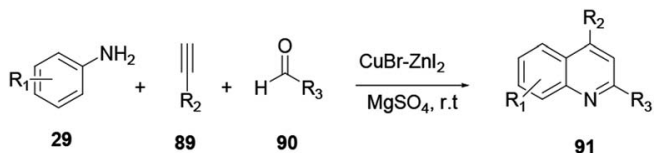

Scheme 24 Copper-zinc combo-catalysed synthesis of quinoline derivatives.

2-Substituted quinoline $\mathbf{9 7}$ is prepared from either 2-aminobenzyl alcohol and alkyne/ketone or 2-aminophenethyl alcohol and aldehyde using an AgOTf catalyst. ${ }^{35}$ Synthetically important heterocyclic anchored quinolines, such as furan, pyrrole and thiophene, can be synthesized via a facile and economic procedure using a silver triflate catalyst in toluene solvent with commercially available precursors and additives, as reported by Xu and co-workers (Scheme 25). Afterwards, the silver triflate catalysed one-step synthesis of quinoline with a broad scope of substrates was reported by $\mathrm{Xu}$ and coworkers. ${ }^{36}$

Multicomponent reactions proceeding using aniline 37, aldehyde 98, and ketones $\mathbf{9 9}$ or $\mathbf{1 0 0}$ reacting in the presence of a silver triflate catalyst provide quinolines 101 or 102 (Scheme 26).

Xie and co-workers reported an effective and suitable protocol for the synthesis of 2-aminoquinoline 104 using an $\mathrm{AgBF}_{4}$ catalyst in DMF solvent. ${ }^{37}$ The reaction proceeds using isothiocyanate 103 under basic and oxidant-free mild reaction conditions to provide good to excellent yields (Scheme 27).

The reaction of commercially available starting materials aniline $\mathbf{1 4}$ and aryl allyl alcohol $\mathbf{1 0 5}$ using a palladium acetate catalyst in DMSO solvent via an oxidative cyclization reaction provides quinoline 106 (Scheme 28). ${ }^{38}$ It does not need to employ any acid, base or additive to promote the reaction. The procedure works with a broad scope of substrates and strong electron-withdrawing substituted materials to furnish moderate to satisfactory yields.

\section{Bioactivities of quinolines}

Both natural products and synthetic compounds which are anchored with a quinoline scaffold have certainly exhibited a broad range of biological or pharmaceutical activities. ${ }^{39,40}$ Among them are antibacterial, ${ }^{41,42}$ antioxidant, ${ }^{43}$ anticancer, ${ }^{44}$ anti-inflammatory, ${ }^{45,46}$ antimalarial, ${ }^{47,48}$ and antifungal activities. ${ }^{49}$

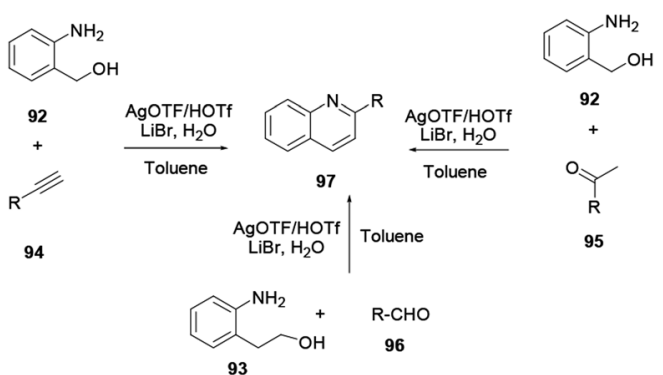

Scheme 25 Silver triflate catalysed synthesis of quinoline derivatives.

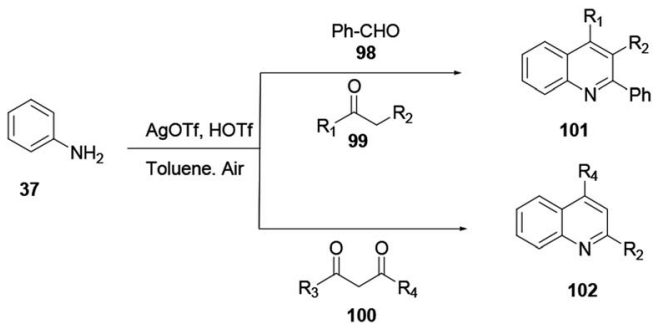

Scheme 26 Silver triflate mediated synthesis of polysubstituted quinolines.

\subsection{Antibacterial activity}

Desai and co-workers reported the synthesis of quinoline derivatives 107, 108 and 109 exhibiting the most powerful antimicrobial activities (Fig. 3). ${ }^{50}$ The synthesized compounds were screened for their potential antibacterial activity on Staphylococcus aureus, Streptococcus pyogenes, Escherichia coli, and Pseudomonas aeruginosa using ampicillin as a standard drug. The recorded results revealed that these compounds have good bacterial activity with minimum inhibition concentrations of $12.5 \mu \mathrm{g} \mathrm{ml} l^{-1}$ and $50 \mu \mathrm{g} \mathrm{ml} l^{-1}$. Furthermore, the authors claim that the potential activity of these compounds is directly associated with the substituent effect on the ring. These three bioactive quinoline derivatives are linked using hydrazone, as reported by Le and co-workers. ${ }^{51}$ Quinoline derivatives with a hydrazone linker 110, 111 and 112 showed good growth inhibition of targeted bacteria.

Furthermore, Fu and co-workers synthesized hybridized quinoline derivative $\mathbf{1 1 3}$ with a piperazine moiety linker and reported that it showed broad-spectrum antibacterial activities on selected bacteria with MIC values of $0.125-8 \mu \mathrm{g} \mathrm{ml}{ }^{-1} .^{52}$

\subsection{Antioxidant activity}

Bazine and co-workers report that quinoline derivative $\mathbf{1 1 4}$ anchored with an $\alpha$-aminophosphate revealed effective antioxidant activity when compared with standard DPPH (Fig. 4). ${ }^{53}$ The authors confirmed that the bioactivity was further modified through the introduction of a phenol ring as a substituent to the quinoline scaffold.

\subsection{Anticancer activity}

Bingul and co-workers synthesized and reported quinoline derivative $\mathbf{1 1 5}$ for anticancer activity against neuroblastoma cells (Fig. 5). The authors revealed that compound 115 showed reasonable anticancer activities against $\mathrm{SH}-\mathrm{SY} 5 \mathrm{Y}$ and Kelly neuroblastoma cell lines and decreased the viability of neurocancer cells with substantial selectivity over normal cells. ${ }^{54}$ Compounds 117 and 118 are quinoline derivatives anchored with

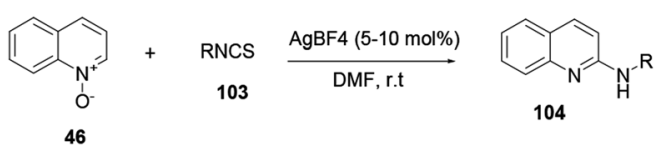

Scheme 27 Silver-catalysed amination of quinoline $\mathrm{N}$-oxides 


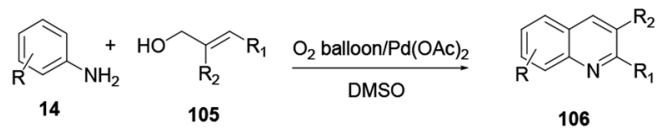

Scheme 28 Palladium-catalysed synthesis of quinoline derivatives.

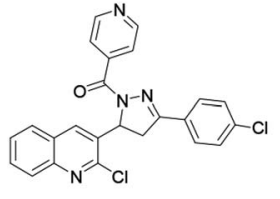

107

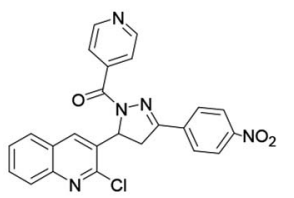

109
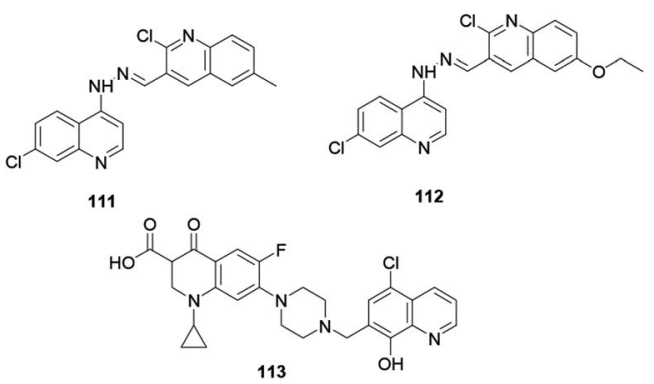

Fig. 3 Chemical structures of antibacterially active quinoline derivatives.

a thiophene moiety, reported by Othman and co-workers. ${ }^{55}$ The above quinolines exhibited powerful anticancer activity against MCF-7 human cancer cells with $\mathrm{IC}_{50}$ values of 38.41 and 28.36 $\mu \mathrm{M}$, respectively. Additionally, Kundu and co-workers reported that quinoline 116 with an imidazole and 1,3,4-oxadiazole exhibited the highest potency for human topoisomerase $1 .^{56}$

\subsection{Anti-inflammatory activity}

Tseng and co-workers synthesized and reported indeno[1,2-c] quinoline derivatives 119 as a potent anti-TB agent, besides being a potent anti-inflammatory agent with low cytotoxicity (Fig. 6). ${ }^{57}$

\subsection{Antileishmanial activity}

Upadhyay and co-workers synthesized and reported that quinoline derivative $\mathbf{1 2 0}$ anchored with a triazole exhibited

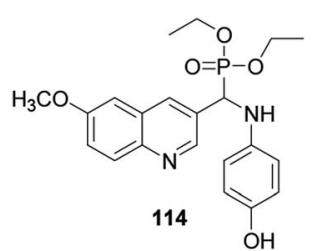

Fig. 4 Chemical structure of an antioxidant active quinoline derivative.

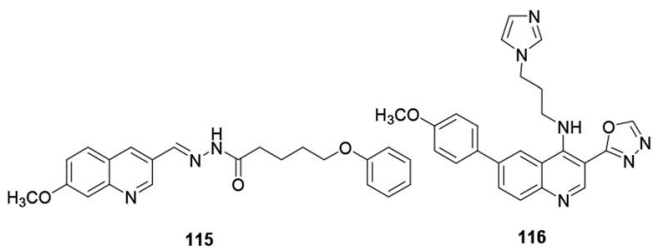

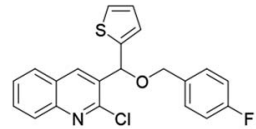

117

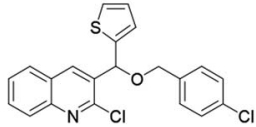

118
Fig. 5 Chemical structures of anticancer active quinoline derivatives.

antileishmanial activity (Fig. 7).$^{58}$ The authors revealed that having a chloro-substituent enhanced the activity of the synthesized compound.

Phosphorated quinoline $\mathbf{1 2 1}$ is a hybrid and potent antileishmanial agent. ${ }^{59}$ Phosphorus hybridized with quinoline is a promising basis for strong antileishmanial activity.

\subsection{Antimalarial activity}

Currently, scholars are exploring how to improve and enhance the antimalarial activity of compounds with a quinoline scaffold. These are mainly synthesized quinoline derivatives hybridized with commercially available and potentially recognized drugs. ${ }^{\mathbf{6 0}}$

Investigators claim that hybridization will result in benefits of cost-effectiveness and minimize the risk of drug-drug interaction. Lombard and co-workers reported quinoline hybridized with artemisinin drug and provided compound 122 (Fig. 8). The hybrid compound exhibited antimalarial activity, although not as much as dihydroartemisinin, but it showed excellent antiplasmodial activity. ${ }^{61}$ The antimalarially active quinolinesulfonamide hybrid derivative 123 was synthesized and reported by Verma and co-workers. ${ }^{62}$ The authors revealed that the hybrid compound exhibited inhibition of the formation of hemozoin.

\subsection{Antifungal activity}

Antifungal active compounds 6-perfluoropropanyl quinoline 124 and 125 were synthesized and reported by Fang and coworkers (Fig. 9). ${ }^{63}$ The synthesized quinoline derivatives exhibited excellent antifungal activity against Pyricularia oryzae. El Shehry and co-workers synthesized and reported an antifungally active pyrazole-quinoline hybrid 126 (Fig. 9). ${ }^{64}$ The

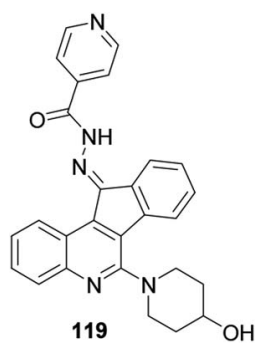

Fig. 6 Chemical structure of anti-inflammatory active quinoline derivative. 

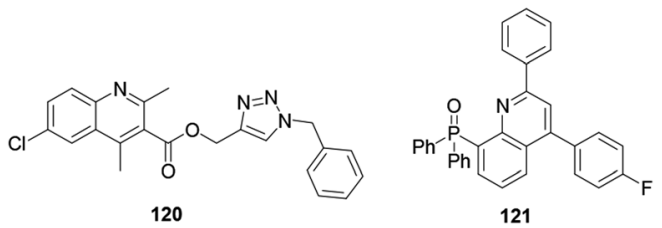

Fig. 7 Chemical structure of antileishmanial active quinoline derivative.

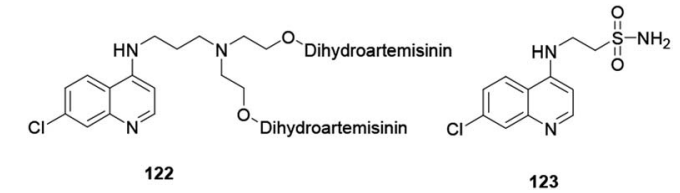

Fig. 8 Chemical structures of hybridized quinolines.
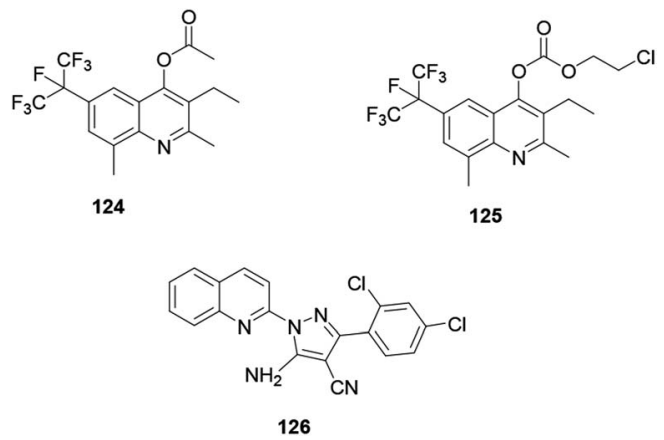

Fig. 9 Chemical structures of antifungal active quinoline derivatives.

synthesized compound exhibited good antifungal activity against the target fungal species.

\section{Conclusions}

In conclusion, numerous quinoline derivatives play a big role in the progress of organic synthesis and applications to medicinal chemistry. Recently, researchers have synthesized hybrid quinoline scaffolds with compounds containing other heterocyclic compounds using different procedures. For example, through transitional metal catalysed or metal-free reactions, ultrasound irradiation, or multicomponent one-pot synthesis, modified named reactions can be instigated to synthesize various noble and effective quinoline derivatives. Generally, from the abovementioned procedures metal-free, ionic liquid and ultrasound irradiation synthesis methodologies meet the requirements of 'green chemistry'.

Until now the synthesis and modification of a quinoline scaffold and investigations to improve its biological and pharmaceutical activities have been continually developing. ${ }^{65,66}$ Nowadays researchers are focused not only on the synthesis of quinoline and its derivatives but also on developing and designing eco-friendly reaction procedures. Typical metal-free, solvent-free, aqueous media and ionic liquid catalysed reactions are green protocols. ${ }^{67}$ Quinoline and its derivatives have exhibited a potentially wide range of applications to treat various kinds of human infections, such as bacterial infections, cancer, malaria and fungal infections. The above-mentioned synthesis protocols based on green synthesis procedures are usually suggested for preparing this noble organic compound and its analogues.

\section{Conflicts of interest}

Article content has no conflict of interest.

\section{References}

1 S. Jain, V. Chandra, P. K. Jain, K. Pathak, D. Pathak and A. Vaidya, Arabian J. Chem., 2019, 12, 4920-4946.

2 P. G. Shobhashana, P. Prasad and M. P. Patel, Heterocycl. Lett., 2017, 7, 819-828.

3 A. Marella, O. P. Tanwar, R. Saha, M. R. Ali, S. Srivastava, M. Akhter, M. Shaquiquzzaman and M. M. Alam, Saudi Pharm. J., 2013, 21, 1-12.

4 T. Jennifer, B. Andrew, K. Stanislaw, W. Ying, C. Manwika and D. Stevan, J. Org. Chem., 2017, 82, 1073-1084.

5 M. F. Mehrjardi, Mini-Rev. Org. Chem., 2017, 14, 187-196.

6 N. Anand, T. Chanda, S. Koley, S. Chowdhury and M. S. Sing, RSC Adv., 2015, 5, 7654-7660.

7 I. Elghamry and Y. Al-Faiyz, Tetrahedron Lett., 2016, 57, 110112.

8 Z. H. Skraup, Monatsh. Chem., 1881, 2, 139-170.

9 G. A. Ramann and B. J. Cowen, Tetrahedron Lett., 2015, 56, 6436-6439.

10 G. A. Ramann and B. J. Cowen, Molecules, 2016, 21, 9861009.

11 R. Sharma, P. Kour and A. Kumar, J. Chem. Sci., 2018, 73, 130-155.

12 A. Aboelnaga and T. H. El-Sayed, Green Chem. Lett. Rev., 2018, 11, 254-263.

13 X. F. Shang, S. L. Morris-Natschke, Y. Q. Liu, X. Guo, X. S. Xu, M. Goto, J. C. Li, J. Y. Zhang and K. H. Lee, Med Res Rev., 2018, 38, 1614-1660.

14 S. Xiao-Fei, L. M. Susan, L. Ying-Qian, G. Xiao, X. Xiao-Shan, G. Masuo, L. Jun-Cai, Y. Guan-Zhou and L. Kuo-Hsiung, Med. Res. Rev., 2018, 38, 775-828.

15 H. Yalgin, D. Luart and C. Len, J. Flow Chem., 2016, 6, 80-85. 16 M. K. Kumawat, P. Parida and D. Chetia, Med. Chem. Res., 2016, 25, 1993-2004.

17 (a) L. Y. Xie, Y. J. Li, J. Qu, Y. Duan, J. Hu, K. J. Liu, Z. Cao and W. M. He, Green Chem., 2017, 19, 5642-5646; (b) Z. Cao, Q. Zhu, Y. W. Lin and W. M. He, Chin. Chem. Lett., 2019, 30, 2132-2138.

18 L. Yamin, Z. Xiaoqiang, W. Zhaoyang, C. Jinhui, M. Chaowei, H. Yongqin and H. Guosheng, RSC Adv., 2015, 5, 8821488217.

19 Q. Gao, S. Liu, X. Wu, J. Zhang and A. Wu, J. Org. Chem., 2015, 80(11), 5984-5991.

20 W. Kun, H. Zhiliang, L. Chao, Z. Heng and L. Aiwen, Chem. Commun., 2015, 51, 2286-2289. 
21 A. S. Kumar, R. A. Kumar, V. Satyanarayana, E. P. Reddy, B. J. M. Reddy, D. N. Kumar, A. Khurana, G. Chandraiah and J. S. Yadav, Nat. Prod. Commun., 2017, 12, 1129-1132.

22 G. J. Nicholas, D. H. Jared, B. C. Emily, M. B. Samer, H. P. Amy, A. P. Julie, J. K. Jacques and G. D. Matthew, Beilstein J. Org. Chem., 2018, 14, 2529-2536.

23 M. Zhu, C. Wang, W. Tang and J. Xiao, Tetrahedron Lett., 2015, 56, 6758-6761.

24 L. Y. Xie, S. Peng, F. Liu, G. R. Chen, W. Xia, X. Yu, W. F. Li, Z. Cao and W. M. He, Org. Chem. Front., 2018, 5, 2604-2609.

25 B. Du, P. Qian, Y. Wang, H. Mei, J. Han and Y. Pan, Org. Lett., 2016, 18, 4144-4147.

26 S. Peng, Y. X. Song, J. Y. He, S. S. Tang, J. X. Tan, Z. Cao, Y. W. Lin and W. M. He, Chin. Chem. Lett., 2019, 30, 22872290.

27 L. Y. Xie, T. G. Fang, J. X. Tan, B. Zhang, Z. Cao, L. H. Yanga and W. M. He, Green Chem., 2019, 21, 3858-3863.

28 X. Xu, Y. Yang, X. Chen, X. Zhang and W. Yi, Org. Biomol. Chem., 2017, 15, 9061-9065.

29 S. Das, D. Maiti and S. D. Sarkar, J. Org. Chem., 2018, 83, 2309-2316.

30 C.-Z. Luo, P. Gandeepan, Y.-C. Wu, W.-C. Chen and C.-H. Cheng, RSC Adv., 2015, 5, 106012-106018.

31 W. Chen, Q. Ding, Z. Nie and Y. Peng, RSC Adv., 2016, 6, 48767-48773.

32 B. S. Prashant, P. B. Sandeep and S. C. Hemantm, Tetrahedron Lett., 2016, 57, 5753-5756.

33 P. Bao, L. Wang, Q. Liu, D. Yang, H. Wang, X. Zhao, H. Yue and W. We, Tetrahedron Lett., 2019, 60, 214-218.

34 R. R. Mondal, S. Khamarui and D. K. Maiti, ACS Omega, 2016, 1, 251-263.

35 X. Xu, X. Zhang, W. Liu, Q. Zhao, Z. Wang, L. Yu and F. Shi, Tetrahedron Lett., 2015, 56, 3790-3792.

$36 \mathrm{X} . \mathrm{Xu}$, W. Liu, Z. Wang, Y. Feng, Y. Yan and X. Zhang, Tetrahedron Lett., 2016, 57, 226-229.

37 L. Y. Xie, S. Peng, L. L. Jiang, X. Peng, W. Xia, X. Yu, X. X. Wang, Z. Cao and W. M. He, Org. Chem. Front., 2019, 6, 167-171.

38 J. Xu, J. Sun, J. Zhao, B. Huang, X. Li and Y. Sun, RSC Adv., 2017, 7, 36242-36245.

39 N. J. P. Subhashini, J. Amanaganti, L. Boddu and P. A. Nagarjuna, J. Chem. Pharm. Res., 2013, 5, 140-147.

40 W. Gao, J. Liu, Y. Jiang and Y. Li, Beilstein J. Org. Chem., 2011, 7, 210-217.

41 N. C. Desai, G. M. Kotadiya and A. R. Trivedi, Bioorg. Med. Chem. Lett., 2014, 24, 3126-3130.

42 R. Vlahov, J. Parushev, P. Nickel and G. Snatzke, J. Pure Appl. Chem. Res., 1990, 7, 1303-1306.

43 B. Vivekanand, K. M. Raj and B. H. M. Mruthyunjayaswamy, J. Mol. Struct., 2015, 1079, 214-224.

44 V. Spanò, B. Parrino, A. Carbone, A. Montalbano, A. Salvador, P. Brun, D. Vedaldi, P. Diana, G. Cirrincione and P. Barraja, Eur. J. Med. Chem., 2015, 102, 334-351.

45 S. A. El-Feky, Z. K. Abd El-Samii, N. A. Osman, J. Lashine, M. A. Kamel and H. K. Thabet, Bioorg. Chem., 2015, 58, 104-116.
46 M. A. Kerry, G. W. Boyd, S. P. Mackay, O. Meth-cohn and L. Platt, J. Chem. Soc., Perkin Trans. 1, 1999, 2315-2321.

47 S. Vandekerckhove and M. D'hooghe, Bioorg. Med. Chem., 2015, 23, 5098-5119.

48 M. A. Lyon, S. Lawrence, D. J. William and Y. A. Jackson, J. Chem. Soc., Perkin Trans. 1, 1999, 437-442.

49 S. Pramilla, S. P. Garg and S. R. Nautiyal, Indian J. Heterocycl. Chem., 1998, 7, 201-204.

50 N. C. Desai, B. Y. Patel and B. P. Dave, Med. Chem. Res., 2017, 26, 109-119.

51 T. D. Le, N. N. Pham and T. C. Nguyen, J. Chem., 2018, 2018, 1-8.

52 H.-G. Fu, Z.-W. Li, X.-X. Hu, S.-Y. Si, X.-F. You, S. Tang, Y.-X. Wang and D.-Q. Song, Molecules, 2019, 24, 548-558.

53 I. Bazine, Z. Cheraiet, R. Bensegueni, C. Bensouici and A. Boukhari, J. Heterocycl. Chem., 2020, 1-11.

54 M. Bingul, O. Tan, C. R. Gardner, S. K. Sutton, G. M. Arndt, G. M. Marshall, B. B. Cheung, N. Kumar and D. S. Black, Molecules, 2016, 21, 916-935.

55 D. I. A. Othman, K. B. Selim, M. A.-A. El-Sayed, A. S. Tantawy, Y. Amen, K. Shimizu, T. Okauchi and M. Kitamura, Bioorg. Med. Chem., 2019, 27, 115026-115038.

56 B. Kundu, S. K. Das, S. P. Chowdhuri, S. Pal, D. Sarkar, A. Ghosh, A. Mukherjee, D. Bhattacharya, B. B. Das and A. Talukdar, J. Med. Chem., 2019, 62, 3428-3446.

57 C.-H. Tseng, C.-W. Tung, C.-H. Wu, C.-C. Tzeng, Y.-H. Chen, T.-L. Hwang and Y.-L. Chen, Molecules, 2017, 22, 1001-1016. 58 A. Upadhyay, P. Kushwaha, S. Gupta, R. P. Dodda, K. Ramalingam, R. Kant, N. Goyal and K. V. Sashidhara, Eur. J. Med. Chem., 2018, 154, 172-181.

59 A. Tejería, Y. P. Pertejo, R. M. Reguera, R. C. Andres, R. B. Fouce, C. Alonso, E. M. Encinas, A. Selas, G. Rubiales and F. Palacios, Eur. J. Med. Chem., 2019, 162, 18-31.

60 X. Nqoro, N. Tobeka and B. A. Aderibigbe, Molecules, 2017, 22, 2268-2290.

61 M. C. Lombard, D. D. N'Da, J. C. Breytenbach, N. I. Kolesnikova, C. T. Van Ba, S. Wein, J. Norman, P. Denti, H. Vial and L. Wiesner, Eur. J. Pharm. Sci., 2012, 47, 834-841.

62 S. Verma, S. Pandey, P. Agarwal, P. Verma, S. Deshpande, J. K. Saxena, K. Srivastava, P. M. S. Chauhan and Y. S. Prabhakar, RSC Adv., 2016, 6, 25584-25593.

63 Y.-M. Fang, R.-R. Zhang, Z.-H. Shen, H.-K. Wu, C.-X. Tan, J.-Q. Weng, T.-M. Xu and X. H. Liua, J. Heterocycl. Chem., 2017, 55, 240-245.

64 M. F. El Shehry, M. M. Ghorab, S. Y. Abbas, E. A. Fayed, S. A. Shedid and Y. A. Ammar, Eur. J. Med. Chem., 2018, 143, 1463-1473.

65 P. Gisbert, M. Albert-Soriano and I. M. Pastor, Eur. J. Org. Chem., 2019, 4928-4940.

66 P. da Silveira, S. Ligia and T. R. A. Vasconcelos, Mini-Rev. Org. Chem., 2019, 16, 602-608.

67 V. F. Batista, D. C. G. A. Pinto and A. M. S. Silva, ACS Sustainable Chem. Eng., 2016, 4, 4064-4078. 